Reportes de Tesis De Posgrado

\title{
Diademas cerebrales para niños con problemas de atención
}

\author{
Hugo Rodríguez
}

Publicado: 21 Septiembre 2016

\begin{abstract}
Resumen
Las terapias de neuro-retroalimentación han demostrado ser útiles para tratar el trastorno de déficit de atención e hiperactividad (TDAH). Para realizar esta terapia, el paciente requiere utilizar una diadema cerebral que se coloca en el cuero cabelludo y/o en los lóbulos de las orejas. Sin embargo su diseño no es apropiado para el uso de niños en edad preescolar que se pueden beneficiar de las terapias de retroalimentación neuronal, en especial aquellos preescolares que tengan problemas de atención. Esta tesis propone diseñar, implementar y evaluar diademas cerebrales adecuadas para preescolares y evaluar su usabilidad y experiencia de uso en comparación con las diademas disponibles en el mercado.
\end{abstract}

Palabras clave: Interfaces cerebrales; Neuroretroalimentación; Diseño de interacción; Atención.

\section{Introducción}

Quizá uno de los mayores descubrimientos de la neurociencia es la caracterización de las funciones neurológicas y mentales como la comunicación química entre las neuronas a través de millones de sinapsis que conectan entre sí a un cerebro humano. Esto ha contribuido al estudio y desarrollo de nuevos métodos y herramientas en la forma de gorras, cascos, y diademas para medir la actividad cerebral con el fin de tener un mejor entendimiento del funcionamiento del cerebro.

Estas diademas han mejorado la accesibilidad de nuevos métodos para mejorar por ejemplo los problemas de atención de niños mediante terapias de neuro-retroalimentación. Sin embargo, los terapeutas y niños encuentran estas diademas difíciles de utilizar y la mayoría las rechazan ya que las perciben muy invasivas.

En esta tesis, se propone utilizar impresoras 3D y sensores para diseñar, implementar y evaluar diademas cerebrales que sean más apropiadas para utilizarse por niños en edad preescolar.

\section{Trabajos relacionados}

En los últimos años existe un crecimiento significativo en cuanto al desarrollo e investigación relacionadas con las Interfaces cerebrales (e.g., Brain-Computer Interfaces o BCIs por sus siglas

\footnotetext{
Rodríguez, $\mathrm{H}$.

Departamento de Ciencias de la Computación

CICESE,

Ensenada, México,

Email: hrodriguez@ cicese.edu.mx
}

en inglés) [4]. Una BCI se define como un sistema basado en computadora que adquiere las señales del cerebro, las analiza y las traduce en comandos que están relacionados con un dispositivo de salida para llevar a cabo una acción deseada [10].

Existen diademas comerciales que utilizan técnicas de electroencefalogramas (EEG) tradicionales que son de bajo costo y que permiten monitorear la actividad cerebral. Por ejemplo, Epoc de Emotiv es una diadema que utiliza 14 Electrodos y 4 referencias, los cuales antes de colocarse se preparan con solución salina para medir la actividad cerebral de los individuos. En contraste, MindWave de NeuroSky y Muse de Interaxon son diademas que utilizan menos electrodos (i.e. Mindwave 1 electrodo y una referencia y Muse 4 electrodos y 3 referencias) y que se pueden utilizar en seco. El trabajo de investigación en esta área ha sido más activo en utilizar las diademas como mecanismos de entrada para adaptar las aplicaciones de atención [6] y meditación o controlar video juegos [8]. Es decir, se han abocado a buscar las "visualizaciones cerebrales" que permitan mantener la atención al niño dentro de la terapia. Sin embargo, existen muy pocos trabajos que estudien y evalúen el diseño de las diademas dadas las características del usuario a utilizarlas.

Respecto al diseño de las diademas. Mihajlović et al. [8] mencionan que estos dispositivos deben satisfacer las necesidades del usuario tales como conveniencia y comodidad. Además hace observaciones como que las diademas o lectores de actividad cerebral que se encuentran actualmente en el mercado son muy pesadas y difíciles de utilizar. Como una solución se propone el uso de diseños ligeros, amigables con el usuario, fáciles de utilizar, con electrodos que hagan un buen contacto con el cuero cabelludo y tengan una distribución adecuada. Por ejemplo, enfatizan que los electrodos deberían adherirse de manera natural al momento de colocar el dispositivo. Ekandem et al. [2] realizaron un estudio para evaluar la ergonomía de las diademas comerciales Mindwave de NeuroSky y Epoc de Emotiv. Los resultados de este estudio indican que Mindwave se considera fácil de colocar pero algo incómodo debido a la presión que ejerce el electrodo que se coloca en la frente y la pinza de referencia que se coloca en la oreja. Por otro lado Epoc fue considerado más cómodo, pero más difícil de colocar; ya que posee más electrodos (14 en total) y se requiere que los electrodos se coloquen con solución salina.

Si bien, en recientes años varios proyectos han estudiado potenciales aplicaciones de BCIs en la práctica diaria; en la literatura no ha sido muy explorado el espacio de diseño para diademas para niños preescolares y únicamente se tiene evidencia del diseño de sistemas que usan EEG para esta población [9]. 


\section{Objetivos de investigación}

Los trabajos relacionados nos presentan una oportunidad para el desarrollo de tecnología adecuada para que los niños preescolares puedan obtener las ventajas de utilizar terapias de neuroretroalimentación. En base a esto se deriva el objetivo general de investigación.

\subsection{Objetivo general}

Diseñar e implementar una diadema cerebral apropiada para leer la actividad cerebral de niños de preescolar y evaluar su usabilidad y experiencia de uso en comparación a las diademas comerciales actualmente disponibles en el mercado.

De este objetivo se desprenden las siguientes preguntas de investigación:

- P1: ¿Cuáles son las características físicas de diseño que debe de tener una diadema cerebral apropiada para niños preescolares?

- P2: ¿Cuántos electrodos y que configuración debe de tener esta diadema para leer de manera apropiada la actividad cerebral de niños preescolares?

- P3: ¿Cuál es la experiencia de uso y usabilidad de la diadema propuesta en comparación con diademas comerciales en niños?

\section{Métodos}

Para lograr los objetivos de este trabajo de investigación se propone la siguiente metodología de trabajo:

Para resolver P1, se realizará un estudio contextual con preescolares para entender sus preferencias e identificar los problemas de usabilidad presentes al utilizar las diademas comerciales. Para capturar esta información, se utilizarán métodos de etnografía rápida y para el análisis de la información se utilizarán técnicas cualitativas. De ser posible, se buscará la opinión de expertos en áreas como diseño de productos y ergonomía. Como resultado espero obtener implicaciones o características de diseño que guíen el diseño de prototipos alternativos de diademas cerebrales apropiadas para niños en edad preescolar.

Para resolver P2, se pretende buscar en la literatura las configuraciones de electrodos existentes y de ellas elegir la más apropiada para capturar la actividad cerebral de los niños preescolares. Una vez elegida la configuración, se realizarán talleres de diseño participativo en donde los niños propondrán bosquejos de potenciales diademas apropiadas para sus necesidades. Estos talleres estarán basados en trabajos en los cuales se integra a los niños a las actividades de diseño. Se les brindan materiales, se les plantea una problemática a solucionar que involucre al dispositivo [1] y se les pone como objetivo el crear algún dispositivo con los materiales proporcionados $[1,3,5]$. Como resultado se propondrán un conjunto de escenarios y prototipos de una diadema apropiada para preescolares. Para el análisis de los datos se utilizarán técnicas de diseño contextual rápido. Para validar los diseños de los prototipos obtenidos, se buscará el apoyo de expertos en diseño de productos y ergonomía con el fin de elegir el mejor para su posterior implementación y evaluación.

Para resolver P3, se implementará alguno de los diseños propuestos y se realizará en un estudio experimental en el laboratorio. Con los resultados obtenidos en este estudio, se comparará el uso del prototipo de la diadema propuesta con los resultados obtenidos del estudio con las diademas comerciales que se realizó para resolver P1. Los métodos de análisis incluyen cuestionarios y observaciones que miden la usabilidad y experiencia de uso de prescolares.

\section{Conclusiones y contribuciones}

Como trabajo a futuro llevaré a cabo todas las etapas planteadas en la metodología. Terminaré de realizar el estudio contextual, para analizar los datos cualitativos obtenidos y después comenzar con el proceso de diseño. Después, desarrollaré un prototipo robusto para poder implementarlo y por ultimo evaluaré la utilidad y el valor que este prototipo ofrece en comparación a las diademas comerciales.

En cuanto a posibles contribuciones, se espera proveer evidencia empírica de que las diademas cerebrales diseñadas son más apropiadas para los niños que las diademas comerciales.

\section{Agradecimientos}

A todas las personas involucradas en esta investigación, a CICESE por proporcionar un espacio de trabajo y materiales, y al CONACyT por los recursos económicos proporcionados.

\section{Referencias}

[1] Derboven, J., Van Mechelen, M., and Slegers, K. Multimodal Analysis in Participatory Design with Children. Proceedings of the 33rd Annual ACM Conference on Human Factors in Computing Systems - CHI '15, ACM Press (2015), 28252828.

[2] Ekandem, J.I., Davis, T. a., Alvarez, I., James, M.T., and Gilbert, J.E. Evaluating the ergonomics of BCI devices for research and experimentation. Ergonomics 55, 5 (2012), 592-598.

[3] Frauenberger, C., Makhaeva, J., and Spiel, K. Designing Smart Objects with Autistic Children. Proceedings of the 2016 CHI Conference on Human Factors in Computing Systems - CHI '16, ACM Press (2016), 130-139.

[4] Hamadicharef, B. Brain-Computer Interface (BCI) literature - A bibliometric study. 10th International Conference on Information Sciences, Signal Processing and their Applications, ISSPA 2010, Isspa (2010), 626-629.

[5] Mann, A., Hinrichs, U., and Quigley, A. Children's Creativity Lab: Creating a 'Pen of the Future.' Proceedings of the 2014 Workshops on Advances in Computer Entertainment Conference - ACE '14 Workshops, ACM Press (2014), 1-9.

[6] Marshall, D., Coyle, D., Wilson, S., and Callaghan, M. Games, gameplay, and BCI: The state of the art. IEEE Transactions on Computational Intelligence and AI in Games 5, 2 (2013), 82-99.

[7] Mason, S.G. and Birch, G.E. A general framework for braincomputer interface design. IEEE Engineering in Medicine and Biology Society 11, 1 (2003), 70-85.

[8] Mihajlovic, V., Grundlehner, B., Vullers, R., and Penders, J. Wearable, Wireless EEG Solutions in Daily Life Applications: What are we Missing? IEEE Journal of Biomedical and Health Informatics 19, 1 (2015), 6-21.

[9] Perego, P., Sironi, R., Lavezzari, R., and Andreoni, G. UserCentered Design for Wearable Neuro-rehabilitation System. Proceedings of the 2015 workshop on Wearable Systems and Applications - WearSys '15, ACM Press (2015), 57-58.

[10] Shih, J.J., Krusienski, D.J., and Wolpaw, J.R. Braincomputer interfaces in medicine. Mayo Clinic Proceedings 87, 3 (2012), 268-279 